\title{
Contexto y evolución de la sostenibilidad en el curriculum de la universidad española
}

\author{
Anna Maria Geli de Ciurana \\ Universitat de Girona, Institut de Recerca Educativa. Girona. España. \\ am.geli@udg.edu \\ ORCID: https://orcid.org/0000-0002-8337-3210 \\ Leslie Mahe Collazo Expósito \\ Universitat de Girona, Institut de Recerca Educativa. Girona. España. \\ leslie.collazo@udg.edu \\ ORCID: https://orcid.org/0000-0003-1267-0143 \\ Ingrid Mulà Pons de Vall \\ Universitat de Girona, Institut de Recerca Educativa. Girona. España. \\ ingrid.mula@udg.edu \\ ORCID: https://orcid.org/0000-0002-5691-1159
}

[Recibido: 12 Noviembre 2018. Revisado: 12 Enero 2019. Aceptado:06 Febrero 2019]

Resumen: En este artículo se exponen los avances y las dificultades de las universidades españolas para incorporar la formación para la sostenibilidad en el curriculum académico de los estudios universitarios, en el marco del Sistema Universitario Español (SUE): las decisiones políticas del gobierno y los claustros universitarios, los cambios estructurales en las instituciones, las alianzas interuniversitarias e internacionales que facilitan el intercambio de experiencias y las líneas de investigación que desarrollan las universidades para incorporar los principios de la sostenibilidad en los programas de todas las titulaciones. El artículo se propone mostrar la responsabilidad de las universidades con la sostenibilidad y apunta algunos retos de futuro.

Palabras clave: Educación para la sostenibilidad, desarrollo sostenible, educación superior, curriculum

\section{The evolution of embedding sustainability in the higher education curriculum in Spain}

Abstract: This paper presents the progress and difficulties of Spanish universities in embedding education for sustainable development in the curriculum, within the context of the Spanish Higher Education System: university political decisions and governance, structural changes of universities, inter-university and international partnerships that facilitate the exchange of experiences, and lines of research carried out by universities that focus on the embedding of sustainability principles in courses and programmes. The article seeks to highlight the responsibility that universities have in addressing sustainable development and points out key future challenges.

Keywords: Education for sustainability, sustainable development, higher education, curriculum

Para citar este artículo: Geli, A. M., Collazo, L. y Mulà, I. (2019). Contexto y evolución de la sostenibilidad en el curriculum de la universidad española. Revista de Educación Ambiental y Sostenibilidad 1(1), 1102. doi: 10.25267/Rev_educ_ambient_sostenibilidad.2019.v1.i1.1102

\section{Introducción}

A comienzo del siglo XXI, diferentes factores, como el cambio climático y las guerras en diferentes puntos del planeta, con el desplazamiento de millones de personas, han convertido la sostenibilidad en un reto fundamental para nuestras generaciones. A lo 
largo del siglo pasado, la industrialización y los nuevos estilos de vida, con la aparición de la sociedad del consumo, provocaron la sobre-explotación de los recursos naturales y la contaminación de las aguas, el suelo y el aire, causando la aparición de problemáticas desconocidas hasta ahora. Se hace necesario impulsar unas relaciones diferentes entre la sociedad y la naturaleza. Esto significa un cambio en el estilo de vida, un cambio en la manera de vivir y en las convicciones y prioridades de las personas. La educación, dirigida a todas las edades y a todos los ámbitos sociales, contribuye a facilitar este cambio. En el momento presente, la Educación para el Desarrollo Sostenible (EDS) se ha convertido en un compromiso con el futuro de la sociedad y del planeta.

Naciones Unidas lidera desde los años 70 del siglo XX las propuestas de educación ambiental con una serie de estudios y documentos que se convierten en referentes mundiales: la Carta de Belgrado, UNESCO (1975); la Conferencia de Tbilisi, UNESCO (1977); el Informe Brundtlland, WCED (1987); la Cumbre de Rio de Janeiro, UNESCO (1992); la Cumbre de Johannesburg, UNESCO (2002); la Década de la Educación para el Desarrollo Sostenible 2005-2014, UNESCO (2005); Rio+20 Treaty On Higher Education, COPERNICUS Alliance (2012); la Conferencia de Aichi-Nagoya, UNESCO (2014a), hasta llegar al Plan de Acción Global (2014b) y la Agenda 2030, NU (2015) con los 17 Objetivos para el desarrollo Sostenible (ODS).

En todos los documentos se plantea la educación de toda la sociedad de manera interdisciplinaria, a través de la educación formal, desde las etapas de educación infantil y obligatoria hasta la educación superior y universitaria, también la educación no formal e informal a lo largo de toda la vida. El importante rol de las universidades en el desafío de la sostenibilidad es reconocido por el conjunto de la sociedad y las organizaciones internacionales. Esto ha inspirado la creación de mandatos específicos para las universidades en las principales declaraciones de medio ambiente y sostenibilidad (Estocolmo del 1972, Río del 1992, Johannesburgo del 2002, Río+20 del 2012, etc.).

A comienzos del milenio, ante la grave situación del sistema, la ONU proclamó el Decenio de la Educación para el Desarrollo Sostenible 2005-2014 (DEDS). Su objetivo fue integrar los principios, valores y prácticas del desarrollo sostenible en todos los aspectos de la educación y el aprendizaje, con la finalidad de fomentar los cambios de comportamiento necesarios para preservar en el futuro la integridad del medio ambiente y la viabilidad de la economía, y para que las generaciones actuales y venideras gocen de justicia social (UNESCO, 2005). De esta forma fueron definidos los principales temas de la sostenibilidad: la biodiversidad, el cambio climático, la reducción del riesgo de desastres naturales, la diversidad cultural, la reducción de la pobreza, igualdad de género, la promoción de la salud, estilos de vida sostenibles, paz y seguridad humana, el agua y las urbanizaciones sostenibles (UNESCO, 2005).

Estos temas generales comenzaron a acelerar aún más la inclusión de los principios de la sostenibilidad en la educación, poniendo la EDS en el punto de mira de la comunidad internacional. En este escenario, en el 2014, la Conferencia Mundial sobre la Educación para el Desarrollo Sostenible de Aichi-Nagoya (Japón) concluyó con un reclamo de acciones urgentes para generalizar la EDS e incluirla en el programa de desarrollo posterior (UNESCO, 2014a). La Declaración de Aichi-Nagoya pidió a todas 
las naciones que pusieran en marcha el Programa de Acción Mundial para la EDS (GAP, por sus siglas en inglés).

Este programa da seguimiento al Decenio de la EDS (2005-2014) y tiene por objeto generar y ampliar la EDS y acelerar el progreso hacia el desarrollo sostenible. Para ello se propone contribuir a la agenda 2030 con dos objetivos fundamentales: reorientar la educación y el aprendizaje para que todos puedan adquirir conocimientos, habilidades, valores y actitudes que los empoderen y les permitan contribuir a un futuro sostenible y fortalecer la educación y el aprendizaje en todas las agendas, programas y actividades que promuevan el desarrollo sostenible (UNESCO, 2015).

El Programa Global Acción de la UNESCO en Educación para el Desarrollo Sostenible (2014b) identifica como una prioridad para los sistemas educativos un cambio total en las instituciones universitarias. La UNESCO pide específicamente reforzar la capacidad y desarrollo profesional de educadores, formadores y otros agentes de transformación para convertirse en facilitadores de la EDS.

Poco después, las Naciones Unidas lanzan la Agenda 2030 que ya venía trabajándose desde la Conferencia de Rio+20 en 2012. La Agenda 2030 propone 17 ODS que abarcan todas las esferas de la vida y del concepto de sostenibilidad (NU, 2015). Este esfuerzo de las Naciones Unidas, que muestra un nivel de concreción sin precedentes, está resultando un marco facilitador de la EDS que promete permitir grandes avances en el reto de la sostenibilidad.

En este momento, la formación para la sostenibilidad en el ámbito universitario toma una especial relevancia por su impacto social en todos los campos de conocimiento. Los graduados universitarios lideran el ámbito laboral y sus convicciones inciden en las decisiones de empresas, entidades e instituciones.

En este contexto cabe recordar que la universidad siempre ha sido un motor de progreso y evolución social, en los países europeos. Su misión docente se ha desarrollado y organizado a lo largo de siglos, caracterizando a la universidad como cuna del saber y promotora de la cultura. La incorporación de la misión investigadora en el siglo XIX, con el modelo de estructura departamental del alemán Von Humboldt, ha añadido a la institución universitaria la capacidad de generar conocimiento en todos los campos del saber e impulsar sus fronteras hacia nuevos retos y desafíos. Como institución educativa transmisora de cultura y como centro de creación de conocimiento, las universidades han asumido, en el siglo $\mathrm{XX}$, su tercera misión de transferencia e innovación, para impulsar políticas y sistemas de mejora de la sociedad y del entorno.

En el marco de estas tres misiones la universidad española ha asumido su compromiso institucional para adoptar políticas que den respuesta a los desafíos que tiene la sociedad en este nuevo milenio. De acuerdo con las líneas marcadas por Naciones Unidas, las universidades han empezado el camino hacia la sostenibilidad en todos los ámbitos de su competencia.

En este artículo se exponen los avances y las dificultades de las universidades españolas para incorporar la formación para la sostenibilidad en el Sistema Universitario Español (SUE): las decisiones políticas, los cambios estructurales, las alianzas interuniversitarias e internacionales y las líneas de investigación que 
orientan la docencia para la sostenibilidad en la formación universitaria. El artículo pretende también mostrar la sensibilidad de las universidades con el reto de la sostenibilidad y el camino que queda por hacer.

\section{Estructuras para incorporar la sostenibilidad en las instituciones universitarias}

A finales del siglo pasado, las universidades españolas crean, de manera autónoma y a partir de iniciativas individuales, las primeras estructuras que permiten impulsar la sostenibilidad desde una perspectiva interdisciplinar y global que abarcan todas las áreas de conocimiento y todas las misiones de la universidad: institutos, oficinas, departamentos y titulaciones dedicados a impulsar las nuevas Ciencias Ambientales. Este concepto aparece en aquellos años y se mantiene y evoluciona hacia el concepto de desarrollo sostenible, citado por primera vez en el Informe Brundland del año 1987, que tomó protagonismo a partir de la Cumbre de Rio en 1992. Casi al mismo tiempo surge el concepto de sostenibilidad, que desarrolla el concepto de desarrollo sostenible y se define como una manera de pensar y de vivir que facilita la convivencia respetuosa con todos los seres vivos y la relación con nuestro planeta.

La mayoría de universidades pusieron en marcha institutos, departamentos y seminarios de medio ambiente, como ámbito de investigación interdisciplinares para abordar las problemáticas ambientales. Las oficinas verdes o servicios técnicos de apoyo a las acciones de sostenibilidad ambiental en todos los ámbitos de la universidad diseñan los planes generales de ambientalización de la universidad, aprobados por los equipos directivos y los claustros universitarios, que marcan las líneas de trabajo y sus objetivos ambientales a corto y medio plazo.

Paralelamente, se diseñan titulaciones y estudios para la formación en ciencias ambientales, específicamente orientadas a la formación de especialistas en el estudio del impacto que la actividad humana genera en nuestro planeta. La sensibilidad hacia estos temas hace que algunas universidades se avancen a las decisiones del Consejo de Universidades y diseñen estudios de grado que se imparten como título propio de la universidad hasta que, poco después, son homologados como título oficial. También se inician programas de postgrado, máster y doctorados en este campo de conocimiento.

Más tarde, las universidades completan la gestión de la sostenibilidad con vicerrectorados o adjuntos a la dirección con competencias específicas en los aspectos ambientales y de sostenibilidad de la institución. A menudo esta competencia se asocia a vicerrectorados de campus o infraestructuras que velan para mejorar las condiciones de sostenibilidad de edificios y campus universitarios.

En el año 2001, en Bolonia (Italia), los estados de la Unión Europea firman los acuerdos para crear el Espacio Europeo de Educación Superior (EEES), lo cual comportó cambios profundos en los estudios del sistema universitario español. En octubre de 2007, se promulga el Real Decreto que marca las directrices para el diseño de los planes de estudios de convergencia europea. Desde este momento, las licenciaturas y diplomaturas se transforman en grados, aparecen los másteres oficiales y, poco después, se organiza el nuevo modelo de doctorado. A partir de la implementación de los nuevos títulos se diseña la formación universitaria centrada en competencias. En estos años, siguiendo las directrices de los nuevos planes de 
estudio, se diseñan las titulaciones a partir de las competencias que deben acreditar los estudiantes para obtener el título correspondiente de grado o máster. En estos planes de estudio, diseñados entre los años 2008 y 2010, las universidades empiezan a incluir la sostenibilidad como competencia transversal, común a todas las titulaciones.

Por otra parte, las universidades disponen de estructuras que se encargan de la formación docente inicial y continua del profesorado universitario de todas las áreas del conocimiento. Con la finalidad de orientar al profesorado en la consecución de las competencias de los nuevos planes de estudios, se imparten cursos y se editan guías y materiales docentes que, en algunas universidades, incluyen indicaciones respecto a la sostenibilidad, como competencia transversal.

Sin embargo, a pesar de todo el proceso iniciado y de las estructuras y documentos creados, debemos señalar dos aspectos que, des de nuestra perspectiva, nos permiten pensar que el proceso hacia la sostenibilidad no ha avanzado de manera eficaz. En primer lugar, son pocos los docentes que han incorporado la competencia de la sostenibilidad en su programa. Y, en segundo lugar, autores como Schumacher (1973) y Tilbury (2011) nos señalan que, a pesar del aumento de los contenidos que se transmiten sobre sostenibilidad, los problemas ecológicos, económicos y sociales siguen creciendo. Por lo tanto, debemos trabajar por una educación que prepare al alumnado para llegar a la profundidad de los fenómenos y los comprometa con la acción. De esta forma podremos mejorar los procesos que permitan avanzar hacia la sostenibilidad en la educación superior y que ésta quede plenamente integrada en el curriculum universitario.

Finalmente, se inicia una tercera línea de estructuras institucionales para incorporar la participación de la sociedad en los proyectos universitarios para la sostenibilidad. En el año 2011 se publica el Libro Blanco de la RSU en España: Responsabilidad Social Universitaria y Desarrollo Sostenible, impulsado por el Ministerio de Educación y coordinado por la Universidad Complutense de Madrid (MEC, 2011), con la participación de profesores y expertos de diversas universidades, empresas y entidades con amplia experiencia en este campo. Esta publicación enlaza las aportaciones de las universidades con la experiencia del entorno social y empresarial y constituye un referente para avanzar en este concepto. Las estructuras de compromiso social o Responsabilidad Social Universitaria (RSU), que, con diferentes formatos, como cátedras, oficinas o programas de RSU, trabajan con entidades sociales, empresas y ONGs. La mayoría de las cátedras de RSU creadas en universidades españolas se articulan en una red interuniversitaria patrocinada por el Banco de Santander. Al mismo tiempo, los consejos sociales de las universidades y las oficinas que conectan la universidad y la empresa también impulsan la Responsabilidad Social Corporativa para desplegar acciones de solidaridad y apoyo social y ambiental en las entidades de su entorno.

En la actualidad, las iniciativas en sostenibilidad tomadas por las universidades entre la última década del siglo XX y la primera del siglo XXI constituyen estructuras plenamente consolidadas que han superado los recortes de la crisis económica de los años 2010-2013 y cuentan con el apoyo institucional y la colaboración de la comunidad universitaria para desarrollar su labor. 


\section{Las alianzas interuniversitarias y partenariados sociales del nuevo milenio}

Pronto se comprendió la necesidad de establecer alianzas que permitieran avanzar en una meta que no puede conseguirse sin la participación del conjunto de la sociedad. Así se han ido creando redes entre universidades, incluyendo también a diversas instituciones que han permitido muchos de los progresos realizados hasta el momento. En los últimos años, también hemos visto como redes surgidas de proyectos comunitarios ganan relevancia a nivel mundial, con la participación de múltiples centros de enseñanza e instituciones. En este trabajo mencionamos algunas de estas redes, con el objetivo de ofrecer una visión del panorama general.

En España, a partir del año 2002, doce universidades, lideradas por la Universidad Autónoma de Madrid, organizan un pequeño grupo de trabajo que inicia sus aportaciones sobre Calidad Ambiental, Desarrollo Sostenible y Prevención de Riesgos (CADEP) bajo la tutela de la Conferencia de Rectores de las Universidades Españolas (CRUE). A este grupo de trabajo, se fueron incorporando universidades públicas y privadas que deseaban ampliar la gestión de sus campus y sus proyectos docentes e investigadores desde la perspectiva de la sostenibilidad.

En el año 2008, con el rector de la Universidad Autónoma de Madrid presidiendo la CRUE y la Universitat de Girona en la presidencia del grupo de trabajo CADEP, el grupo pasa a ser una de las diez Comisiones Sectoriales estables de la Conferencia de Rectores, quienes presentan las Directrices para la introducción de la Sostenibilidad en el Curriculum (CADEP-CRUE, 2012). A partir de 2014, bajo la presidencia de la Universidad de Alcalá de Henares, toma el nombre de CRUE-Sostenibilidad. Actualmente, participan en esta comisión sectorial la mayoría de universidades españolas, todas ellas comprometidas con el desarrollo sostenible.

A principios de 2015 nace la Red Española para el Desarrollo Sostenible (REDS), red nacional de SDSN en España. Su misión es movilizar y sensibilizar a la sociedad española, a las instituciones públicas y al mundo corporativo para que conozcan de manera más rigurosa y comprometida los ODS, así como favorecer su incorporación a futuras políticas, al ámbito empresarial y en el comportamiento de la sociedad en general. La principal finalidad de la REDS es activar a las universidades, los centros de investigación, las empresas y la sociedad civil para la resolución de problemas prácticos sobre desarrollo sostenible. La REDS centra su actividad en cuatro áreas prioritarias para España: energía, agua, biodiversidad y género.

En el contexto iberoamericano, la Organización Internacional de Universidades por el Desarrollo Sostenible y el Medio Ambiente (OIUDSMA) fue precursora de las redes ambientales en la educación superior. La OIUDSMA pretendió, desde su creación en 1995, actuar como una red de instituciones universitarias que tendrían entre sus objetivos prioritarios el desarrollo de programas docentes e investigadores en el campo del Medio Ambiente y el Desarrollo Sostenible. Sentó las bases de las primeras incursiones de muchas instituciones de educación superior al ámbito de la sostenibilidad y jugó un papel inductor de otras experiencias exitosas como la conformación de la red Alianza de Redes Iberoamericanas de Universidades en Sustentabilidad y Ambiente (ARIUSA) (Rosúa y Serrano, 2016). En España tuvo un gran impacto en el trabajo realizado por las universidades de Granada y Valencia. 
A nivel europeo, un gran número de rectores de las universidades españolas firmaron la carta de COPERNICUS en el 1993 y, más adelante, en 2012, el tratado Rio+20 Treaty on Higher Education. Ambos documentos despliegan una visión estratégica de la sostenibilidad en la universidad y buscan el compromiso de las universidades para promoverla en todos sus ámbitos de actuación.

A nivel internacional, la Global University Network for Innovation (GUNi) es una red presidida por la Asociación Catalana de Universidades Públicas (ACUP) y cuenta con oficinas regionales en Asia y el Pacífico, América Latina y el Caribe, África SubSahariana, los países árabes y Europa y Norteamérica (EEUU y Canadá). Desde su creación, la misión de la GUNi ha sido la de reforzar el rol de la educación superior en la sociedad contribuyendo a la renovación de las visiones y políticas alrededor del mundo bajo una visión de servicio público, relevancia y responsabilidad social. Desde 2011, esta red a incorporado la sostenibilidad en su campo de actuación generando documentos y organizando congresos internacionales donde han participado de forma activa un gran nombre de universidades españolas.

Las redes también han evolucionado de forma acorde con los principios de la sostenibilidad, como es el caso de su carácter holístico, lo cual permite maximizar su potencial de crear alianzas y abrir las puertas a nuevas formas de cooperación entre la sociedad civil, las organizaciones públicas y privadas, incluyendo a todas las vías de educación formal y no formal. Esta tendencia es totalmente coherente con el ODS 17, que se propone fortalecer la alianza mundial para el desarrollo sostenible, partiendo de la premisa de que el trabajo conjunto es la base del éxito.

Un ejemplo destacado es el de la red de transición hacia la sostenibilidad TransitionNetwork que es un movimiento impulsado por la sociedad civil, que comenzó en el año 2005 y no ha dejado de crecer. La red inspira, promueve y guía a las comunidades en sus múltiples formas, como pueblos, ciudades, universidades y escuelas para poner en práctica acciones que permitan enfrentar los grandes desafíos locales y globales (Transition Network, 2018).

Para ello fomentan una cultura solidaria, participativa y emprendedora, bajo los principios de la economía circular y otras iniciativas, siendo creativos, capacitándose y tejiendo redes de conexión y apoyo. Transition Network se ha extendido a más de 50 países, acumulando ya 946 iniciativas alrededor del mundo, entre ellas algunas procedentes de universidades europeas como la Universidad de Edimburgo o la Universidad de St Andrews. En España, cuenta con 16 iniciativas y se ha impartido talleres en centros como el el Centro Nacional de Educación Ambiental (CENEAM), la Universidad Pompeu Fabra, la Universitat Politècnica de Catalunya o la Universidad de Santiago de Compostela, en las que se han comenzado a realizar investigaciones relacionadas con el tema.

\section{Los modelos educativos y la investigación en educación para la sostenibilidad}

Mientras las universidades avanzan creando estructuras transversales que facilitan la incorporación de la sostenibilidad en los campus, diseñan titulaciones con competencias específicas y establecen redes y alianzas interuniversitarias para la sostenibilidad, surge el reto de incorporar la sensibilidad y los principios de la sostenibilidad a toda la formación disciplinar. 
Son numerosos los autores que nos recuerdan la importancia de la investigación educativa rigurosa y contrastada. En el ámbito de la educación para la sostenibilidad, debemos reconocer que la investigación llegó a la educación después de la acción. Es decir, la EDS se inició a partir del voluntarismo y el compromiso personal del profesorado, y ha sido en los últimos veinte años cuando se han planteado investigaciones serias para estudiar y contrastar el resultado de los modelos metodológicos aplicados en la formación universitaria. Mayer (1998) habla de la educación ambiental como un caballo de Troya que entra en el campo de la educación cambiando los modos tradicionales de la enseñanza.

En el contexto actual, podemos situar la formación ambiental para la sostenibilidad en el marco de dos grandes paradigmas: el paradigma de la complejidad, que nos permite interpretar las interacciones del medio natural y social como un sistema de sistemas, con las consecuencias de imprevisibilidad que esto conlleva (Junyent, Bonil y Pujol, 2010) y el paradigma constructivista del aprendizaje, que define el proceso de enseñanza-aprendizaje como un proceso individual de construcción personal y singular del conocimiento, con capacidad de cambiar el pensamiento y la vida de las persones (Bruner, 2001).

Cuando Lucas (1972) definía tres posibles enfoques de la educación ambiental: en el medio, sobre el medio y para el medio, establecía un marco para la incipiente educación ambiental y abría el camino de la investigación educativa para la sostenibilidad. En estos años, el proceso de enseñanza-aprendizaje del medio natural ha evolucionado, desde los modelos clásicos de aprendizaje tradicional y aprendizaje por descubrimiento, a modelos educativos de aprendizaje constructivista con metodologías activas de aprendizaje, basadas en la reflexión personal, el espíritu crítico y la participación de los estudiantes como auténticos protagonistas en la construcción de su conocimiento (Collazo y Geli, 2017). En estos modelos, el profesor pasa a tener una función mediadora y facilitadora del aprendizaje, que se orienta directamente a la realidad y a la acción.

No se trata de aprender sólo para saber sino para saber hacer. La incorporación en las programaciones docentes del concepto competencia para definir los tipos de aprendizajes que se espera que los estudiantes consigan conduce a una nueva forma de enseñar. Este concepto, que procedía del mundo laboral, desplaza el concepto tradicional de aprender conocimientos por el del aprendizaje de capacidades para actuar, basadas en conocimientos, procedimientos, actitudes y valores (Lambrechts, Mulà, Ceulemans y Molderez, 2013).

En este contexto, se desarrollan proyectos de investigación educativa que se proponen incorporar al curriculum universitario los principios de la sostenibilidad de manera transversal. El proyecto ALFA-2000 Ambientalización Curricular en Educación Superior desarrollado por la red ACES define un modelo curricular, el modelo ACES, que favorece la incorporación efectiva de la sostenibilidad en la docencia universitaria (Arbat y Geli, 2002). Este estudio sugiere que para avanzar en la incorporación de la sostenibilidad en el currículo hay que adoptar un compromiso global que facilite planteamientos integrales en la gestión y la docencia del centro educativo (facultades, escuelas o institutos) (Junyent, Geli y Sanchez, 2003). Estos planteamientos concretan fundamentalmente en los siguientes aspectos (figura 1): 


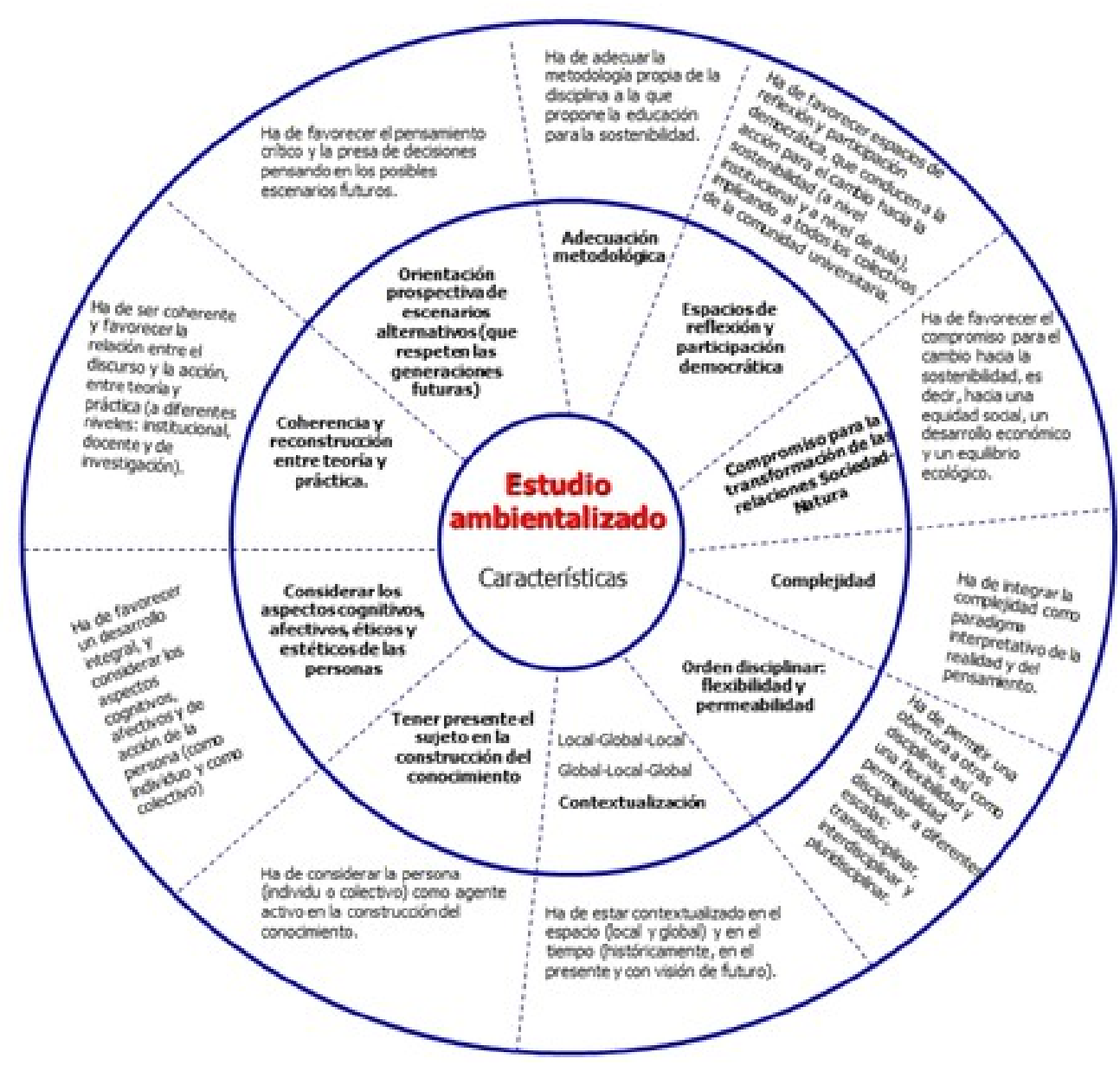

Figura 1. Diagrama Modelo ACES. Fuente: Junyent, Geli y Arbat (2003, p. 38)

Tomar decisiones políticas en el conjunto de la institución:

- Generar espacios necesarios para la participación democrática de los diversos estamentos internos y externos en la definición de las estrategias dirigidas a la sostenibilidad.

- Favorecer el compromiso para el cambio y la innovación en el ámbito de la equidad social, el desarrollo económico y el equilibrio ecológico.

- Adoptar un enfoque institucional global para el desarrollo profesional continuo en sostenibilidad.

Desarrollar procesos de análisis y reflexión para:

- Impulsar la reflexión crítica y ética sobre los escenarios de futuro en las dimensiones socioculturales, ecológicas y económicas del medio.

- Fomentar el desarrollo integral y la participación activa de las personas en la co-construcción de buenas prácticas para el desarrollo sostenible.

- Asegurar la coherencia entre el discurso y la acción a todos los niveles: docencia, organización y gestión institucional. 
Promover cambios imprescindibles en el diseño curricular para impulsar:

- La adecuación de los contenidos, metodologías y prácticas que contribuyen a desplegar competencias para la sostenibilidad.

- La permeabilidad y la flexibilidad disciplinarias.

La contextualización de los temas en el espacio y el tiempo. Estas características del modelo ACES de ambientalización curricular fueron diseñadas en la reunión de la red celebrada en la Universidad Politécnica de Hamburgo (Tutech) en el año 2001 y tuvieron una influencia perceptible en el documento Directrices para la introducción de la Sostenibilidad en el Currículo aprobado por el plenario de rectores españoles en el año 2005 y actualizado en 2012.

Los Ministros de Medio Ambiente de la CEPE-Comisión de Economía de las Naciones Unidas para Europa hicieron en 2003 un llamamiento para incluir la EDS en todos los niveles educativos, desde la educación infantil hasta la educación superior. Este mismo año se puso en marcha el proyecto europeo Comenius 2.1 CSCT (2003), (por sus siglas en inglés: Currículo, desarrollo Sostenible, Competencias, formación Docente) hasta 2007, liderado por la Katholieke Hogeschool Leuven (Bélgica), con 15 socios de 8 países diferentes. En el proyecto se desarrolló un marco para la formación inicial y continua del profesorado, basado en competencias para el desarrollo sostenible. El marco puede adaptarse al contexto local de las instituciones, hace hincapié en el enfoque interdisciplinario y tiene en cuenta los aspectos científicos, económicos, sociales, políticos, culturales y éticos del desarrollo sostenible.

En los últimos años, han sido numerosos los proyectos de investigación desarrollados, con financiación pública, por las universidades españolas que impulsan la incorporación de la sostenibilidad en todos los ámbitos universitarios y en concreto se han planteado líneas de investigación y tesis doctorales para responder al desafío de la formación para la sostenibilidad de los estudiantes universitarios en todas las titulaciones. Las universidades de Cádiz, Granada, Salamanca, Complutense de Madrid, Córdoba, Santiago de Compostela, Valencia, Autónoma de Madrid, Girona, etc. siguen aportando innovaciones que sugieren nuevas metodologías y técnicas para mejorar el aprendizaje de los principios de la sostenibilidad y vincularlos a la práctica profesional de los titulados universitarios en todos los campos

Actualmente la Universidad Politécnica de Catalunya coordina un proyecto, con financiación estatal, en el que participan nueve universidades españolas y que lleva por título Educación e innovación social para la sostenibilidad. Formación en las Universidades españolas de profesionales como agentes de cambio para afrontar los retos de la sociedad (EDINSOST). Este proyecto ha generado encuestas para recoger datos del alumnado y profesorado de las instituciones participantes y propone aportar instrumentos formativos que contribuyan a la formación del profesorado universitario (Sánchez et al., 2017)

También la Universidad de Valencia, la Universidad Nacional de Educación a Distancia y la Universidad Complutense de Madrid, desarrollan un proyecto conjunto, con financiación estatal, para mejorar la formación del profesorado universitario en el ámbito de la sostenibilidad que incluye aportaciones en el campo Competencias para la Sostenibilidad en los estudios universitario, la evaluación de los 
conocimientos, las estrategias docentes y la práctica profesional. Las propuestas metodológicas que impulsan la participación de los estudiantes en la docencia universitaria, se materializan en técnicas concretas de aprendizaje, algunas bien conocidas desde hace tiempo, pero que se están incorporando lentamente en la formación universitaria: aprendizaje basado en problemas, aprendizaje orientado a proyectos, aprendizaje-servicio, TIC y redes sociales, procesos de co-creación, etc. Estos últimos se están utilizando en la actualidad para diseñar escenarios de futuro sostenibles, incluyendo a todos los actores de la sociedad (Collazo, Terradellas, Geli y Benito, 2017).

Por sus especiales características el aprendizaje-servicio se recomienda específicamente para la incorporación de los principios de la sostenibilidad en el currículo universitario puesto que facilita la acción inmediata y la aplicación de los conocimientos adquiridos a la práctica profesional de los titulados. La Comisión sectorial CRUE-Sostenibilidad elaboró un documento de recomendaciones para esta técnica de enseñanza-aprendizaje que muchas universidades aplican a la formación de los estudiantes (CRUE-Sostenibilidad, 2015).

\section{Formación del profesorado universitario}

Varios autores justifican que el éxito de la formación para la sostenibilidad de los titulados universitarios está condicionado por la disposición y el compromiso del profesorado de las universidades (Kapitulcinová et al., 2015). El profesorado, incluso aquellos docentes ya comprometidos, necesita el espacio y tiempo para reflexionar sobre cómo reorientar su práctica docente hacia la sostenibilidad (Cebrián, 2018). El documento Rio+20 Treaty on Higher Education, elaborado por la red COPERNICUS Alliance (2012), coincide con estas afirmaciones y señala la importancia del desarrollo profesional en sostenibilidad del profesorado universitario, así como su autonomía para tomar las decisiones más adecuadas sobre los conocimientos y las metodologías docentes de cada materia y de cada titulación. Reconociendo la necesidad de avanzar en esta dirección, la CEPE creó un grupo de expertos que en 2012 publicaron un marco de competencias de los educadores en EDS (CEPE, 2012). En este estudio, las competencias de los educadores giran en torno a tres ejes:

- El enfoque holístico, que integra reflexión y práctica.

- El planteamiento transformador de la persona educadora.

- La capacidad de la persona educadora de materializar la transformación.

El documento de la CEPE aporta un enfoque valioso para el desarrollo profesional del profesorado universitario que se plantea incorporar los objetivos del desarrollo sostenible en sus disciplinas. Sin embargo, hay que reconocer que, aún hoy, este enfoque académico es poco valorado por las universidades. En general, el profesorado tiene pocos incentivos y poco apoyo para incorporar estos aspectos en su actividad docente. La prioridad tradicional de la excelencia académica de las disciplinas universitarias deja poco margen para la valoración de los perfiles docentes orientados a la transformación de la sociedad.

En algunas instituciones como, por ejemplo, la Universidad de Granada, Universidad Rey Juan Carlos, Universitat Internacional de Catalunya, Universidad del País Vasco, Universitat Politècnica de Catalunya, Universidad de las Palmas de Gran Canaria o 
Universidad Politécnica de Madrid se han organizado seminarios y sesiones de formación específicas en EDS dirigidas al profesorado universitario y al alumnado en formatos presenciales y virtuales. A nivel estatal, los encuentros de investigación que se celebran, en los últimos quince años, en las instalaciones del CENEAM en Valsaín (Segovia) y sus publicaciones son también un foro que permite conocer y compartir experiencias y proyectos en el campo de la sostenibilidad, como representa Martínez Huerta (2014). Aunque estas iniciativas han generado interés en el campo de la EDS, suelen ser formaciones puntuales que no permiten al profesorado desarrollar las competencias necesarias para transformar el curriculum hacia la sostenibilidad.

La formación del profesorado universitario como agente de cambio, con la incorporación de competencias específicas de sostenibilidad en su desarrollo profesional, se trabajó de forma substancial en el proyecto europeo University Educators for Sustainable Development (UE4SD), coordinado por la COPERNICUS Alliance y en el que participaron 53 universidades de 33 países europeos. En España, participaron la Universidad Autónoma de Madrid, la Universitat de Girona, la Universitat Autònoma de Barcelona, la Universidad del País Vasco y la Universidad de Granada. Todas ellas colaboraron en diseñar y realizar una prueba piloto para implementar la Academy for Education for Sustainable Development in Higher Education un programa destinado a impulsar cambios a nivel institucional para mejorar la formación en educación para el desarrollo sostenible del profesorado universitario. Esta iniciativa de desarrollo profesional se ha celebrado a nivel internacional por su carácter innovador y se ha reproducido en Colombia y Perú con el apoyo de las cinco universidades españolas participantes en el proyecto UE4SD. A pesar de esto, el programa no ha continuado en España, reduciendo el impacto que éste pudiese haber tenido a nivel nacional.

Actualmente, el grupo de trabajo en sostenibilización curricular de la CRUE está trabajando en la elaboración de un curso a distancia en sostenibilidad para el PAS y PDI que se ofrecerá de forma gratuita en todas las universidades españolas interesadas. El curso integrará el cuestionario APROSOS, elaborado en colaboración por diversas universidades del grupo de la CRUE (ver CRUE, 2017). Esta encuesta, que tiene como objeto ayudar al profesorado a diagnosticar su grado de conocimiento y compromiso personal con la sostenibilidad. Tiene un carácter propedéutico, ya que la evaluación de las propias capacidades tiene como objetivo orientar el aprendizaje hacia la sostenibilidad.

El curso que elaborará la CRUE significa un gran avance para cubrir un vacío existente a nivel nacional, pero las universidades no pueden limitarse a esto sino que deberán, paralelamente, impulsar sus propias estrategias para motivar y formar a su profesorado. Tal y como apuntan Mulà et al. (2017), la formación para la sostenibilidad comporta la implementación de programas que acompañen al profesorado durante un periodo largo de tiempo a repensar las prioridades curriculares, los objetivos y metodologías docentes.

Más concretamente implica: 
Tabla 1. Cambios necesarios incorporar la sostenibilidad en el curriculum a partir de la formación del profesorado. Fuente: Adaptación de Mulà et al. (2017)

\begin{tabular}{|c|c|}
\hline Pasar de... & a... \\
\hline $\begin{array}{l}\text { Realizar talleres individuales de } \\
\text { desarrollo profesional en EDS }\end{array}$ & $\begin{array}{l}\text { Ofrecer oportunidades de desarrollo profesional } \\
\text { continuo y mentoría para involucrar al docente } \\
\text { activamente en un proceso de reflexión crítica y } \\
\text { acción }\end{array}$ \\
\hline $\begin{array}{l}\text { Utilizar métodos basados en el cambio } \\
\text { personal }\end{array}$ & $\begin{array}{l}\text { Utilizar métodos que apoyan el aprendizaje social } \\
\text { y el cambio }\end{array}$ \\
\hline Formar solo al profesorado & $\begin{array}{l}\text { Formar al personal administrativo y los equipos } \\
\text { directivos }\end{array}$ \\
\hline $\begin{array}{l}\text { Desarrollar la EDS como una disciplina o } \\
\text { especialización }\end{array}$ & $\begin{array}{l}\text { Superar la especialización mediante el cruce de } \\
\text { disciplinas y el trabajo colaborativo }\end{array}$ \\
\hline Fortalecer las identidades académicas & Desarrollar nuevas identidades académicas \\
\hline Integrar la EDS en el curriculum & $\begin{array}{l}\text { Impulsar la innovación del curriculum a partir de } \\
\text { los principios y procesos relacionados con la EDS }\end{array}$ \\
\hline Trasformar la práctica docente & $\begin{array}{l}\text { Promover el cambio institucional y el aprendizaje } \\
\text { organizacional }\end{array}$ \\
\hline $\begin{array}{l}\text { Mejorar la experiencia de aprendizaje } \\
\text { del alumnado }\end{array}$ & $\begin{array}{l}\text { Potenciar el aprendizaje en todos los niveles de la } \\
\text { institución }\end{array}$ \\
\hline \multicolumn{2}{|c|}{$\begin{array}{l}\text { Evaluar los procesos y los resultados para trabajar de forma efectiva la } \\
\text { sostenibilidad en el curriculum }\end{array}$} \\
\hline $\begin{array}{l}\text { Muchas universidades españolas } \\
\text { institucionales en sostenibilidad c } \\
\text { incrementar su transparencia y rend } \\
\text { trabajo de evaluación de la sostenibilic } \\
\text { para evaluar la integración de soste } \\
\text { realizó un estudio para evaluar su } \\
\text { participantes (ver CRUE, 2011). Un gr } \\
\text { participa en el ranquin Green Metrics } \\
\text { que ha ganado mucha fuerza a nive } \\
\text { compartir sus esfuerzos y buenas pr } \\
\text { medir y comparar su nivel de integrac } \\
\text { y cambio climático, gestión de resic } \\
\text { investigación (Green Metrics, 2017) } \\
\text { universidades de } 88 \text { países de to } \\
\text { instituciones, } 5 \text { de las cuales se situar } \\
\text { mundial. Estas son la Universidad de } \\
\text { Universidad Rey Juan Carlos, la Univer } \\
\text { de Madrid. Rencientemente, el Time } \\
\text { ránquines de universiades más c }\end{array}$ & $\begin{array}{l}\text { han empezado a evaluar sus esfuerzos } \\
\text { on la voluntad de mejorar sus procesos, } \\
\text { ir cuentas a la sociedad. En } 2011 \text {, el grupo de } \\
\text { dad de la CRUE definió una serie de indicadores } \\
\text { anibilidad ambiental a nivel institucional y se } \\
\text { grado de cumplimiento en } 31 \text { universidades } \\
\text { ran número de instituciones españolas también } \\
\text {, desarrollado por la Universidad de Indonesia } \\
\text { el global ya que permite a las universidades } \\
\text { rácticas en temas de sostenibilidad, así como } \\
\text { ción en ámbitos como: infraestructuras, energía } \\
\text { duos, uso del agua, movilidad y educación e } \\
\text { D. En la edición de } 2017 \text { participaron } 619 \\
\text { do el mundo. En España, participaron } 28 \\
\text { on en las primeras } 100 \text { posiciones del ranquin } \\
\text { Alcalá, la Universitat Autònoma de Barcelona, la } \\
\text { rsidad de La Coruña y la Universidad Autónoma } \\
\text { o Higher, conocido por la elaboración de los } \\
\text { onocidos mundialmente ha anunciado que } \\
\text { versidades focalizado en la implementación de }\end{array}$ \\
\hline
\end{tabular}


Aunque todas estas iniciativas demuestran un firme compromiso por parte de las universidades españolas a seguir aprendiendo y mejorando en el ámbito de la sostenibilidad, son pocas las que están teniendo un impacto a nivel curricular. Los programas de evaluación más establecidos y con carácter continuo como el Green Metrics se centra sobre todo a medir las actuaciones de las universidades en relación a la gestión ambiental. Los indicadores en el ámbito de la educación e investigación para la sostenibilidad se limitan a cuantificar el número de cursos y publicaciones en este campo sin profundizar en el proceso de enseñanza-aprendizaje que promueve la EDS.

Más recientemente, se ha puesto de relieve la necesidad de vincular la sostenibilidad con los sistemas de evaluación de la calidad para asegurar una implementación más efectiva, productiva y continuada en el tiempo, y poniendo acento a los procesos de aprendizaje. A nivel internacional existen pocas iniciativas que hayan afrontado este reto, pero éstas están abriendo nuevas vías para trabajar y evaluar la sostenibilidad de forma más estratégica en las universidades. En este sentido, destaca el estudio realizado por la Universitat Autónoma de Barcelona encargado por la Agencia de Calidad de la Educación Superior de Andorra donde se propone un marco para incorporar los ODS en el sistema de evaluación de la calidad de las instituciones de educación superior de Andorra (ver Mulà y Junyent, 2017). A raíz de este estudio, se está desarrollando un nuevo proyecto financiado por la Asociación Internacional de Agencias de Calidad de Educación Superior (INQAAHE) que pretende definir unos indicadores de calidad para evaluar la sostenibilidad en los territorios de la Comunidad de Aragón y de Andorra. Estos trabajos demuestran el creciente interés por parte de las agencias de calidad de considerar la sostenibilidad como un elemento de calidad en los sistemas universitarios e integrarla en los procesos de acreditación institucional y de programas. Es una oportunidad única para reconocer el trabajo de las universidades y profesorado que trabaja en este campo y animar aquellos que todavía no han empezado a andar en esta dirección.

\section{Conclusiones y retos de futuro}

Los avances para integrar la sostenibilidad en el SUE han sido notables, principalmente en relación a la gestión del campus, la responsabilidad social y la investigación. A pesar de disponer de estrategias y estructuras destinadas a promover la sostenibilidad en todos los ámbitos de la universidad, la innovación curricular en este campo ha sido menos efectiva y con un impacto débil a nivel institucional (Benayas, Marcén, Alba y Gutiérrez, 2017).

En la mayoría de universidades españolas, la EDS se ha trabajado gracias al entusiasmo de unos pocos docentes comprometidos a incorporar los valores y principios del desarrollo sostenible en sus materias. En algunas instituciones se han creado nuevas asignaturas o diseñado programas especializados en desarrollo sostenible, destinados, especialmente, a alumnado interesado en perseguir una carrera profesional en este campo. Su incorporación en el curriculum, por tanto, se ha realizado de forma fragmentada y con carácter optativo, sin alterar de forma significativa la diversidad de programas que se ofrecen desde las universidades.

Los programas internacionales y las relaciones de las universidades con otras universidades y organizaciones han permitido abrir nuevas vías para trabajar la 
sostenibilidad en el curriculum, pero sobre todo a presionar a las universidades a enfrentarse a este reto de forma estratégica y transversal. Actualmente, está claro que la sostenibilidad no puede ser una realidad sin el compromiso institucional y la participación de toda la comunidad universitaria. Esto es un reto importante para la universidad que necesita de un mayor liderazgo por parte de los equipos rectores y directivos (Scott, Tilbury, Sharp y Deane, 2012). Éstos deben desplegar una visión estratégica y ser capaces de facilitar un proceso de cambio largo, complejo y muchas veces con resultados imprevisibles (UNESCO, 2014c).

En España, la CRUE ha demostrado un claro compromiso y liderazgo en el campo de la sostenibilidad en la educación superior. Su trabajo ha tenido un fuerte impacto en las universidades de todo el estado y como facilitadora de este proceso debe continuar su labor para sumar esfuerzos y conectar a las universidades a esta nueva necesidad social. Este artículo ha puesto de manifiesto que queda mucho camino por recorrer en cuanto a la formación en sostenibilidad. En este sentido, pensamos que la CRUE, por su carácter influyente y dinamizador, juega un papel especialmente importante en la reorientación del curriculum hacia la sostenibilidad. Una línea importante a seguir será la de la formación de los equipos directivos, PDI y PAS en liderazgo para la sostenibilidad para poder garantizar una gestión de la sostenibilidad más integral y estratégica que conecte los diferentes esfuerzos institucionales en relación a la sostenibilidad y ponga mayor foco a los procesos de enseñanzaaprendizaje. Por otro lado, las instituciones deberán detallar sus estrategias en relación a la sostenibilidad y crear sus propios programas de formación a todos los niveles. Finalmente, se espera que el interés en la sostenibilidad por parte de las agencias de calidad siga creciendo y esto dé un nuevo impulso para convencer a las universidades de la necesidad de desarrollar una visión y un plan de acción para integrar el desarrollo sostenible en sus procesos de calidad.

\section{Referencias bibliográficas}

Arbat, E. y Geli, A. M. (2002). Ambientalización Curricular de los Estudios Superiores. Aspectos Ambientales de las Universidades. Girona; Universidad de Girona-Red ACES. ISBN: 84-8458-173-X,

M. Barañano (coordinadora) et al. (Comisión Técnica Nacional de RSU; Anna M. Geli) (2011). La responsabilidad social de la universidad y el desarrollo sostenible. Madrid: Ministerio de Educación. ISBN: 978-84-369-5227-8. Deposito legal: M-47.071-2011,

Benayas, J., Marcén, C., Alba, D. y Gutiérrez, J. M. (2017). .Educación para la sostenibilidad en España. Reflexiones y propuestas Madrid: Fundación Alternativas y Red Española para el Desarrollo Sostenible.

Bruner, J. (2001). El proceso mental en el aprendizaje. Madrid: Ed. Narcea. ISBN: 97884-277-1369-7

CADEP-CRUE (2012). Directrices para la introducción de la Sostenibilidad en el Curriculum.

Cebrián, G. (2018). The I3E Model for Embedding Education for Sustainability within Higher Education Institutions. Environmental Education Research, 24(2), 153171 
CEPE (2012). Learning for the future: competences in Education for Sustainable Development. Ginebra: CEPE.

Collazo, L. M. y Geli, A. M. (2017). Avanzar en la educación para la sostenibilidad: combinación de metodologías para trabajar el pensamiento crítico y autónomo, la reflexión y la capacidad de transformación del sistema. Revista Iberoamericana de Educación, 73(1), 131-154.

Collazo, L., Terradellas Piferrer, M. R., Geli, A. M. y Benito, H. (2017). Formación del Profesorado Universitario, mediante procesos de Co-creación, para incidir en la competencia transversal de la Sostenibilidad. Actas del VII Congreso Universidad y Cooperación al Desarrollo: 'La universidad y los Objetivos de Desarrollo Sostenible'. (pp. 554-564). Madrid: Universidad Autónoma de Madrid y Solana e Hijos, S.A. ISBN: 978-84-8344-570-9.

Comenius

2.1

CSCT

(2003).

https://ensi.org/global/downloads/Publications/303/CSCT

\%20Handbook_11_01_08.pdf

COPERNICUS Alliance (2012). Rio +20 Treaty On Higher Education.

CRUE (2011). Evaluación de las políticas universitarias de sostenibilidad como facilitadoras para el desarrollo de los campus de excelencia internacional.

CRUE (2017). Autodiagnóstico del profesorado en sostenibilización curricular. APROSOS.

CRUE-Sostenibilidad (2015). Institucionalización del Aprendizaje-Servicio como estrategia docente en el marco de la Responsabilidad Social Universitaria para la promoción de la Sostenibilidad.

Geli, A. M., Junyent, M. y Sánchez, S. (2003). Ambientalización curricular de los estudios superiores Diagnóstico de la Ambientalización Curricular de los Estudios Superiores. Girona: Universitat de Girona - Red ACES. ISBN: 84-8458154-3.

Geli, A. M., Junyent, M. y Sánchez, S. (2004). Ambientalización curricular de los estudios superiores Diagnóstico de la Ambientalización Curricular de los Estudios Superiores. Acciones de intervención y balance final del proyecto ACES. Girona: Universitat de Girona - Red ACES. ISBN: 84-8458-202-Z

Geli, A. M., Junyent, M., Medir, R. M. y Padilla, F. (2006). La ambientalización curricular de la enseñanza obligatoria: una propuesta de definición, caracterización y estrategias. Colección monografías Universitarias, 7 Barcelona: Departamento de Medio Ambiente, Generalitat de Cataluña.

Green Metrics (2017). UI GreenMetric World University Ranking Background of the ranking.

Junyent, M., Geli, A. M. y Arbat, E.. (2003). Ambientalización curricular de los estudios superiores. Proceso de caracterización de la Ambientalización Curricular de los Estudios Superiores. Girona: Univ. de Girona - Red ACES. ISBN: 84-8458-190-X.

Junyent, M., Bonil, J. y Pujol, R. M. (2010). Educación para la Sostenibilidad desde la perspectiva de la complejidad. Revista EUREKA sobre Enseñanza y Divulgación de las Ciencias, 7, № Extraordinario 2010, 198-215. 
Kapitulcinová, D., Dlouhá, J., Ryan, A., Dlouhý, J., Barton, A., Mader, M., Tilbury, D., Mula, I., Benayas, J., Alba, D., Mader, C., Michelsen, G. y Vintar Mally, K. (Eds.). Leading Practice Publication: Professional Development of University Educators on Education for Sustainable Development in European Countries. Praga: Charles University of Prague.

Lambrechts, W., Mulà, I., Ceulemans, K. y Molderez, I. (2013). The integration of competences for sustainable development in higher education: an analysis of bachelor programs in management. Journal of Cleaner Production, 48, 65-73.

Lucas, A. (1972). Environment and environmental education: conceptual issues and curriculum implications. Tesis Doctoral, Graduate School of Ohio State University.

Martínez Huerta, J. (2014). Lecciones de una DECAD. La firma del mes. Carpeta Informativa, CENEAM. Abril 2014, 7-15.

Mayer, M. (1998). Educación ambiental: de la acción a la investigación. Enseñanza de las Ciencias, 16(2), 217-231

MEC (2011). La responsabilidad social de la universidad y el desarrollo sostenible. Libro Blanco. Madrid: Secretaría General Técnica. Centro de Publicaciones. Ministerio de Educación.

Mulà, I., Tilbury, D., Ryan, A., Mader, M., Dlouhá, J., Mader, C., Benayas, J., Dlouhý, J. y Alba, D. (2017). Catalysing change for sustainability in higher education: a review of professional development initiatives for university educators. International Journal of Sustainability in Higher Education, 18(5), 798-820.

Mulà, I. y Junyent, M. (2017). La qualitat de l'ensenyament superior d'Andorra i els Objectius de Desenvolupament Sostenible: una proposta d'estàndards i directrius d'avaluació de la qualitat. Andorra la Vella: Agència de Qualitat de l'Ensenyament Superior d'Andorra.

Naciones Unidas (2015). Transforming our world: the 2030 Agenda for Sustainable Development.

Rosúa Campos, J. L. y Serrano Bernardo, F. (2016). La Organización Internacional de Universidades por el Desarrollo Sostenible y el Medio Ambiente (OIUDSMA): un precursor de las redes ambientales en la educación superior iberoamericana. AMBIENS. Revista Iberoamericana Universitaria en Ambiente, Sociedad y Sustentabilidad. 1(2), 49-76.

Sánchez, F., Segalàs, J., Cabré, J., Climent, J., López, D., Martín, C. y Vidal, E. (2017). El proyecto EDINSOST: inclusión de los ODS en la educación superior. Revista española de desarrollo y cooperación, Novembre 2017, 41, 67-81.

Schumacher, E. F. (1973). Small is beautiful: a study of Economics as if people mattered. London: Blond and Briggs.

Scott, G., Tilbury, D., Sharp, L. y Deane, E. (2012). Turnaround leadership for sustainability in higher education. Sydney: Australian Office of Learning and Teaching.

Tilbury, D. (2011). Education for sustainable development: an expert review of processes and learning. París: UNESCO. 
Transition Network (2016). Movimiento de comunidades para reimaginar $y$ reconstruir nuestro mundo.

UE4SD (2016). UE4SD online platform of resources.

UNESCO (1975). La Carta de Belgrado: Una Estructura Global para la Educación Ambiental. Belgrado: UNESCO.

UNESCO (1992). Declaración de Rio sobre el medio ambiente y el desarrollo. Rio de Janeiro, Brasil: UNESCO.

UNESCO (1997). La Conferencia Intergubernamental sobre Educación Ambiental. Tbilisi, URSS: UNESCO.

UNESCO (2002). Declaración de Johannesburgo sobre el Desarrollo Sostenible. Johannesburgo, Sudáfrica: UNESCO.

UNESCO (2005). United Nations Decade of Education for Sustainable Development (2005-2014): International Implementation Scheme. Paris: UNESCO.

UNESCO (2014a). Declaración de Aichi-Nagoya sobre la Educación para el Desarrollo Sostenible. Aichi-Nagoya, Japan: UNESCO.

UNESCO (2014b). UNESCO Roadmap for implementing the Global Action Programme on Education for Sustainable Development. Paris: UNESCO.

UNESCO (2014c). Shaping the future we want: UN Decade of Education for Sustainable Development final report. Paris: UNESCO.

World Commision on Environment And Development (WCED) (1987). Our Common Future (Brundtlland Report). United Nations. 